\title{
Retinal macroaneurysm: natural history and guidelines for treatment
}

\author{
M N ABDEL-KHALEK AND J RICHARDSON \\ From Sunderland Eye Infirmary, Sunderland
}

SUMmaRY This report describes the features and clinical significance of retinal macroaneurysm. Two groups can be defined: those with acute aneurysmal decompensation, Group I; and those with chronic aneurysmal decompensation, Group II. Haemorrhage is the main feature of the former group and exudates of the latter. Systemic and local associations are noted, and guidelines for treatment by phototherapy are discussed.

Although the lesions have been observed since 1808,' Robertson ${ }^{2}$ in 1973 first applied the term 'macroaneurysm' (MA) to retinal arterial lesions with the following characteristic features: (1) a saccular or fusiform ('blow-out' or 'cuffed's) arterial swelling; (2) sited on the first three orders of the retinal arterial tree; (3) found mainly at arterial bifurcations (similar to aneurysms affecting the cerebral circulation).

Correspondence to $\mathrm{Mr} \mathrm{M} \mathrm{N}$ Abdel-Khalek, FRCSEd, Eye Infirmary, Queen Alexandra Road, Sunderland SR2 9HP.
In this report we analyse a series of 19 patients with retinal MAs who were followed up for periods ranging from six months to 10 years. In general, symptoms arose from the sequelae of aneurysmal decompensation. Patients with acute aneurysmal decompensation had intraocular haemorrhage of varying degree (group I, Table 1) and those with chronic decompensation an exudative response (group II, Table 2).

Table 1 Group I: acute aneurysmal decompensation (haemorrhagic)

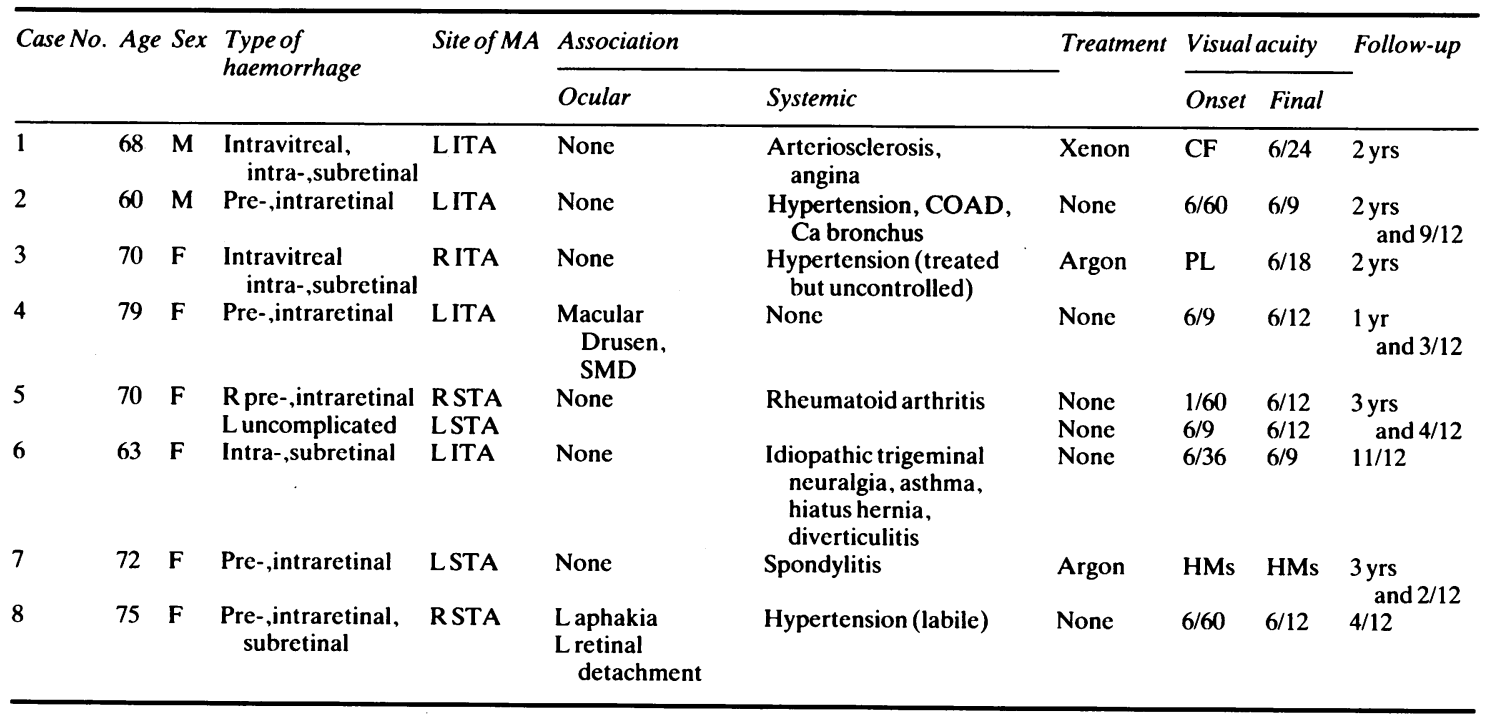

ITA = inferior temporal artery. STA =superior temporal artery. SMD=senile macular degeneration.

$\mathrm{COAD}=$ chronic obstructive airways disease. 
Table 2 Group II: chronic aneurysmal decompensation (exudative)

\begin{tabular}{|c|c|c|c|c|c|c|c|c|c|c|}
\hline \multirow[t]{2}{*}{ Case No. } & \multirow[t]{2}{*}{ Age } & \multirow[t]{2}{*}{ Sex } & \multirow{2}{*}{$\begin{array}{l}\text { Type of } \\
\text { exudate }\end{array}$} & \multirow[t]{2}{*}{ Site of $M A$} & \multicolumn{2}{|l|}{ Association } & \multirow[t]{2}{*}{ Treatment } & \multicolumn{2}{|c|}{ Visual acuity } & \multirow[t]{2}{*}{ Follow-up } \\
\hline & & & & & Ocular & Systemic & & Onset & Final & \\
\hline 1 & 75 & $\mathbf{F}$ & Perianeurysmal & LSTA & None & $\begin{array}{l}\text { Arteriosclerosis, stroke } \\
\text { hypertension, arthritis }\end{array}$ & Clofibrate & $6 / 18$ & $6 / 18$ & $\begin{array}{l}4 \text { yrs, } \\
\text { died }\end{array}$ \\
\hline 2 & 70 & $\mathbf{F}$ & Perianeurysmal & RSTA & Old Choroiditis & $\begin{array}{l}\text { Untreated pernicious } \\
\text { anaemia }\end{array}$ & Xenon & $6 / 12$ & $6 / 12$ & $17 / 12$ \\
\hline 3 & 77 & $\mathbf{F}$ & Perianeurysmal & RSTA & None & Angina & Clofibrate & $6 / 24$ & $6 / 9$ & $\begin{array}{l}18 / 12 \\
\text { died }\end{array}$ \\
\hline 4 & 69 & $\mathbf{F}$ & Perianeurysmal & R STA & None & None & Xenon $\times 5$ & $6 / 9$ & $6 / 9$ & $11 \mathrm{yrs}$ \\
\hline 5 & 72 & $\mathbf{F}$ & $\begin{array}{l}\text { Perianeurysmal, } \\
\text { macular oedema }\end{array}$ & R STA & None & Hypertension, stroke & Clofibrate & $6 / 18$ & $6 / 9$ & $\begin{array}{l}15 / 12 \\
\text { died }\end{array}$ \\
\hline 6 & 74 & $\mathbf{F}$ & Perianeurysmal & LSTA & SMD & Hypertension (untreated) & Xenon & $6 / 18$ & $6 / 18$ & $6 \mathrm{yrs}$ \\
\hline 7 & 60 & $\mathbf{M}$ & $\begin{array}{l}\text { Perianeurysmal, } \\
\text { macular oedema }\end{array}$ & $\begin{array}{l}\text { R ITA } \\
\text { LSTA } \\
\text { LSNA }\end{array}$ & $\begin{array}{c}\text { Bilateral } \\
\text { chronic } \\
\text { uveitis }\end{array}$ & Hypertension (untreated) & Argon & $\begin{array}{l}6 / 36 \\
6 / 36\end{array}$ & $\begin{array}{l}6 / 36 \\
6 / 36\end{array}$ & $\begin{array}{l}2 \text { yrs } \\
\text { and } 6 / 12\end{array}$ \\
\hline 8 & 52 & $\mathbf{F}$ & Perianeurysmal & LITA & None & None & Argon $\times 2$ & $6 / 9$ & $6 / 9$ & $\begin{array}{l}2 \text { yrs } \\
\text { and } 10 / 12\end{array}$ \\
\hline 9 & 61 & $\mathbf{M}$ & Macular exudates & $\begin{array}{l}\text { R STA } \\
\text { R ITA }\end{array}$ & Coats' disease & None & $\begin{array}{l}\text { Xenon } \\
\text { Argon }\end{array}$ & $6 / 24$ & $6 / 60$ & $1 \mathrm{yr}$ \\
\hline 10 & 73 & $\mathbf{F}$ & $\begin{array}{l}\text { Perianeurysmal } \\
\text { macular exudates }\end{array}$ & RSTA & $\begin{array}{l}\text { R STA } \\
\text { A/V occlusion }\end{array}$ & Hypertension & None & $6 / 60$ & $6 / 60$ & $\begin{array}{l}2 \text { yrs } \\
5 / 12 \text { died }\end{array}$ \\
\hline 11 & 73 & $\mathbf{M}$ & $\begin{array}{l}\text { Macular oedema } \\
\text { and exudates }\end{array}$ & RSTA & $\begin{array}{l}\text { R STA } \\
\text { A/V occlusion }\end{array}$ & Hypertension & None & $6 / 12$ & $6 / 6$ & $3 / 12$ \\
\hline
\end{tabular}

STA = Superior temporal artery. ITA =inferior temporal artery. SNA =superior nasal artery.

$\mathrm{SMD}=$ senile macular degeneration. $\mathrm{A} / \mathrm{V}=$ arteriovenous.

\section{Patients and methods}

GROUP I: ACUTE ANEURYSMAL DECOMPENSATION In this group intraocular haemorrhage of variable extent was encountered. When the haemorrhage was substantial we saw preretinal, subhyaloid or intravitreal bleeding. Less florid haemorrhage produced localised intraretinal bleeding.

\section{GROUP II: CHRONIC ANEURYSMAL}

DECOMPENSATION

In this group prolonged abnormal leakage of plasma constituents across the aneurysmal wall led to the accumulation of yellow perianeurysmal intraretinal exudates with or without related macular exudate. A few showed macular exudate only. In cases with abundant circumaneurysmal exudate a high rate of transmural leakage seems likely. Scanty perianeurysmal exudate suggests a low leakage rate.

Of the 19 patients reviewed 17 had unilateral MAs and two had bilateral MAs.

\section{METHODS}

Ophthalmic examination included biomicroscopy, funduscopy, and intravenous fluorescein angiography repeated on various occasions during the follow-up period. In nine patients phototherapy (either xenon arc or argon laser) was used to achieve closure of the MA. Photocoagulation was applied initially in a circular perianeurysmal fashion, using the lowest level of energy necessary to achieve greywhite retinal burns, and then direct to the aneurysm at the same intensity. Only in two cases was it necessary to repeat the treatment. Treatment was

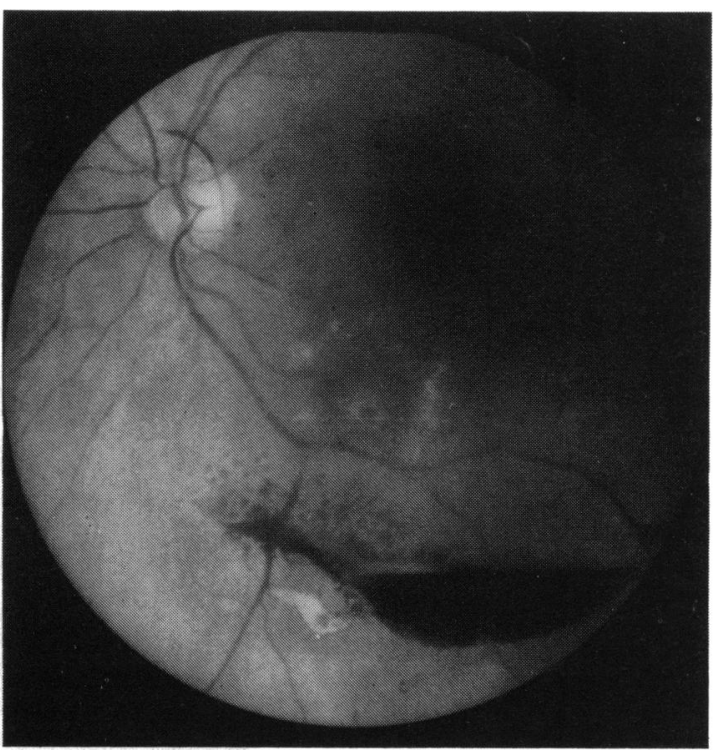

Fig. 1 Preretinal haemorrhage being absorbed, revealing an $M A$ with surrounding exudate. Below that there are pigment clumping and subretinal scarring on the nasal side of the preretinal haemorrhage. 
Fig. 2 Late-phase angiogram.

The MA is of the blow-out type. The cap of perianeurysmal hypofluorescence is clear and may indicate mural thickening. This constant angiographic finding correlates well with the pale aneurysmal halo observed ophthalmoscopically.

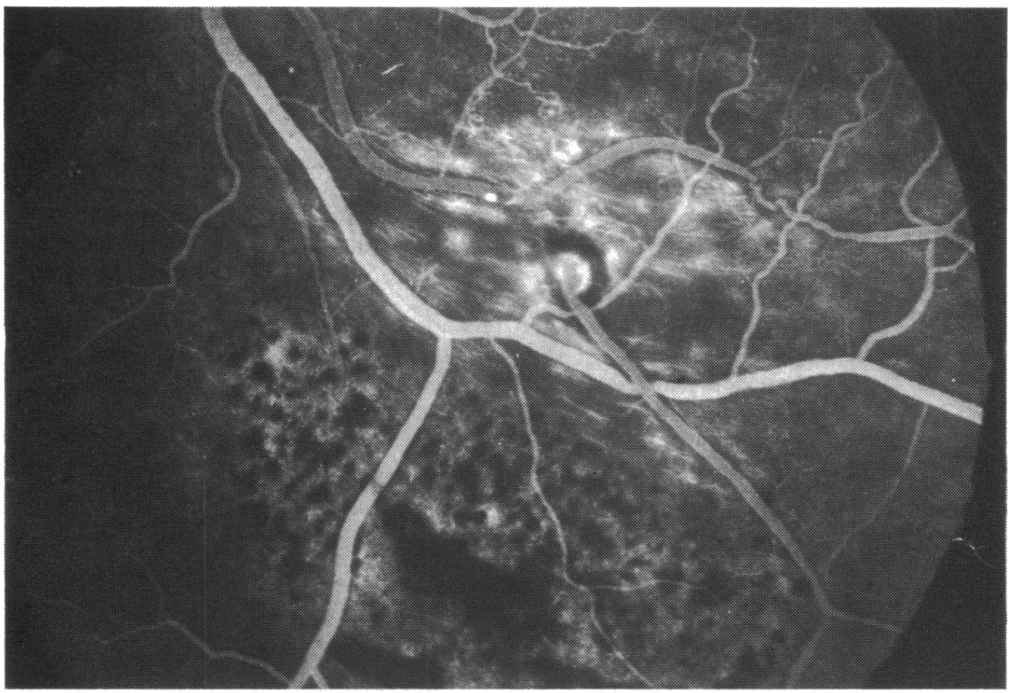

given when macular function was threatened by progressive retinal exudation or by rebleeding.

\section{Results}

\section{GROUP I}

Eight of our patients presented with sudden loss of vision due to haemorrhage from presumed aneurysmal rupture. The haemorrhage ranged in volume from a small, perianeurysmal, intraretinal bleed to a large combined vitroretinal haemorrhage.

The sequence of events following aneurysmal rupture varied. Although the arterial perforation may close, leaving the aneurysm intact, the lesion remained haemodynamically unstable and was pre- disposed to rebleed or allow transmission of tissue fluid across its walls (Fig. 1). Fluorescein angiography might then display the MA with associated vascular anomalies (Fig. 2). In this case aneurysmal closure is called for and can be achieved by direct phototherapy. Fig. 3 shows the angiographic appearance six months after one treatment with xenon arc phototherapy with closure of the MA and characteristic Z-shaped kink at the site of the former aneurysm. More commonly, Z-shaped kinks developed spontaneously after aneurysmal rupture and were apparently stable haemodynamically. They required no active therapeutic intervention. It is likely that many MAs rupture and close spontaneously and go unrecognised as the causative lesion
Fig. 3 In early venous phase the postcoagulative arterial stenosis at site of treatment is displayed. Perianeurysmal capillary meshwork remains showing attenuation. Vascular kink developing (square). Intravascular hyperfluorescent ring has disappeared, suggesting closure of endothelial defect.

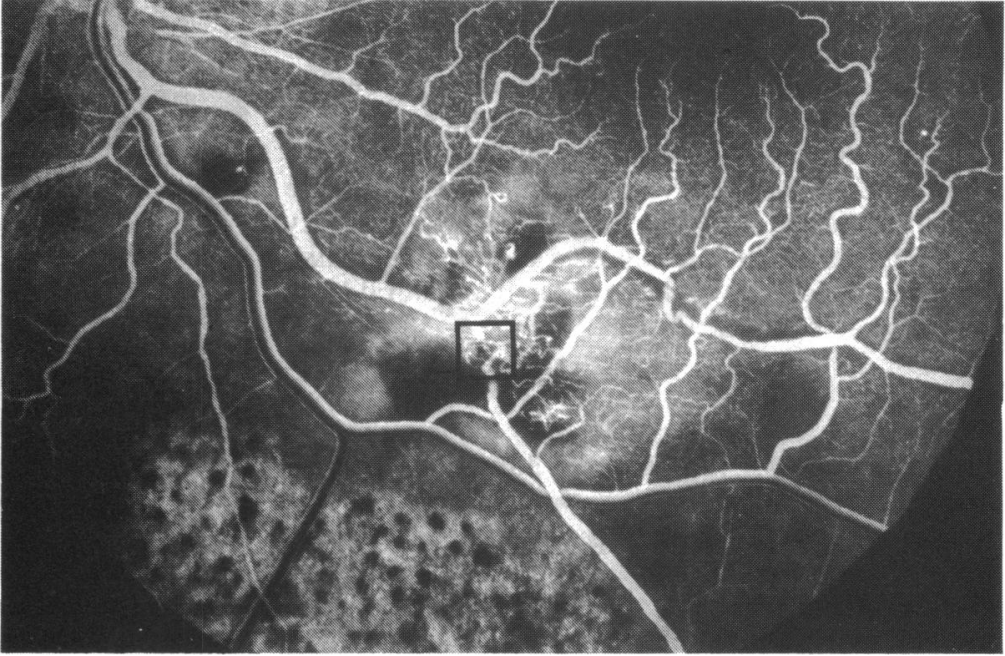




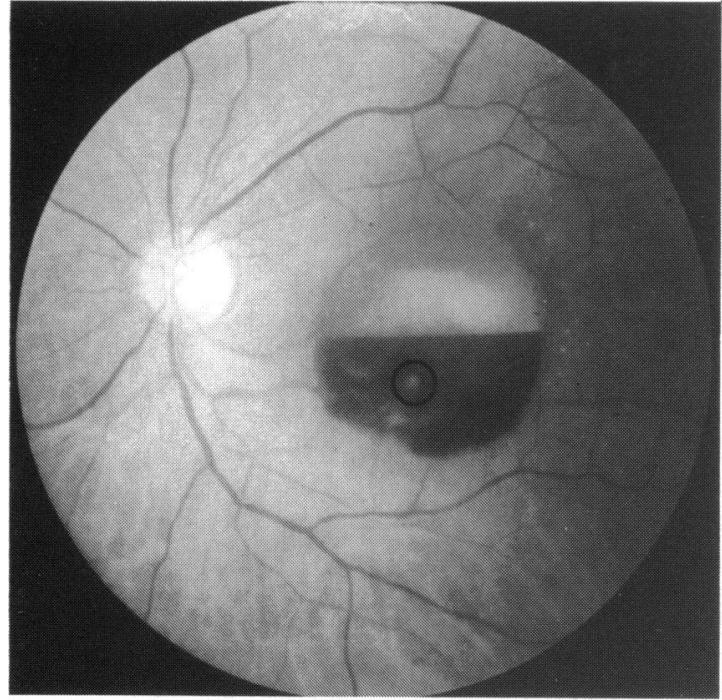

Fig. 4a Preretinal haemorrhage with a fluid level. The segment of the artery harbouring the $M A$ is partly seen through the haemorrhage, and the site of the MA is just visible (circle).

in an episode of intraocular haemorrhage (Figs. $4 a, b)$.

In three cases in which the haemorrhage was only preretinal the MA resolved untreated, with no significant ocular sequelae other than a pathognomonic Z-shaped kink on the offending arteriole marking the site of the former aneurysm. Cases that presented with intraretinal haemorrhage tended to develop a characteristic yellowish-grey saddle-shaped plaque centred on the site of the MA, with surrounding exudate. In such cases the arteriole bearing the MA often went on to become heavily sheathed (Fig. 5). This saddle-shaped plaque is a characteristic posthaemorrhagic lesion and when seen later may cause diagnostic difficulty if the prior retinal haemorrhage was unrecognised.

Those MAs (three of eight group I cases) producing combined pre, intra-, and subretinal haemorrhage may require phototherapy to halt posthaemorrhagic transmural exudation or forestall further bleeding (Fig. 3).

G ROU P II

Eleven cases presented with blurring of vision due to exudation from a retinal MA. Six of these had localised perianeurysmal exudates (Fig. 6), and three had in addition macular exudation. The remaining two had exudates distant from the MA (Fig. 7). Six group II patients had phototherapy to avert macular damage from intraretinal exudate and oedema. Closure of the MA was achieved in four cases by one treatment session only. The other two caes ( 4 and 8 in Table 2) had very large MAs which required more than one treatment session. Closure of the aneurysm was associated with kink formation and gradual exudate dispersal.

In five cases macular function was not threatened and phototherapy was unnecessary. Three of these were seen early in the series and were treated with clofibrate. Spontaneous closure occurred in one, while in the other two exudate formation never became severe.

Two patients had MAs associated with arteriovenous occlusions. In case 10 photocoagulation was planned, as the MA threatened macular function by generating profuse exudate, but the patient died before treatment could be carried out. In case 11

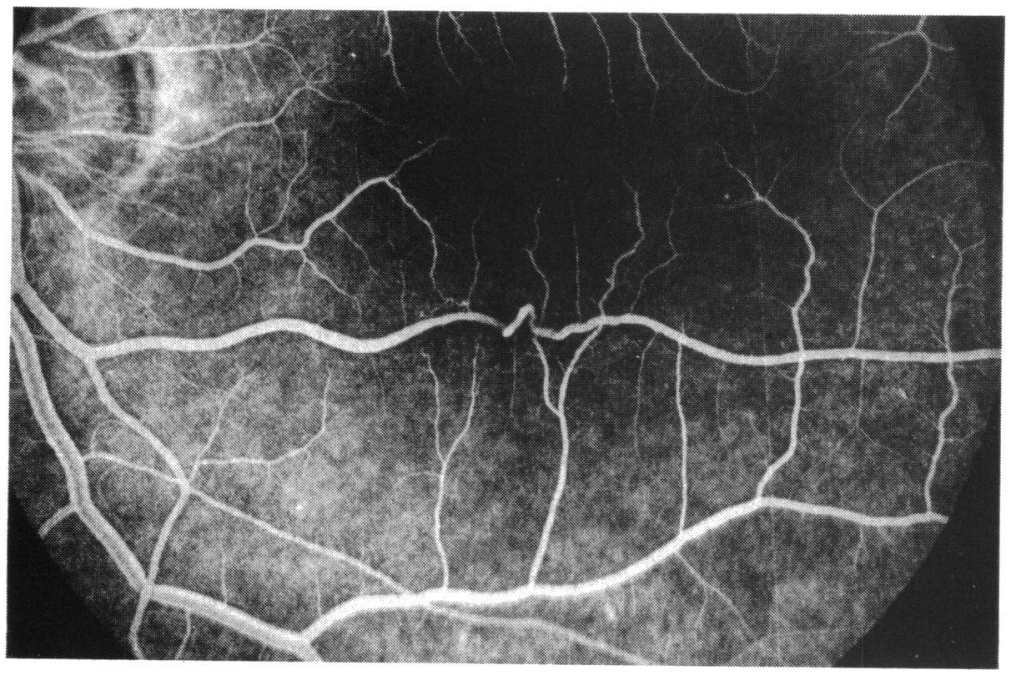

Fig. 4b Nineteen months after the haemorrhage, fluorescein angiogram showing Z-shaped kink at the site of the $M A$ with attenuation of arterial calibre distal to it. 
Fig. 5 Four months after haemorrhage, magnified photograph of the plaque. This shows scattered clumps of pigment resulting from intraretinal haemorrhage and a pigmentary border to the plaque from haemorrhagic disruption of the retinal pigment epithelium (RPE). Note marked arterial sheathing.

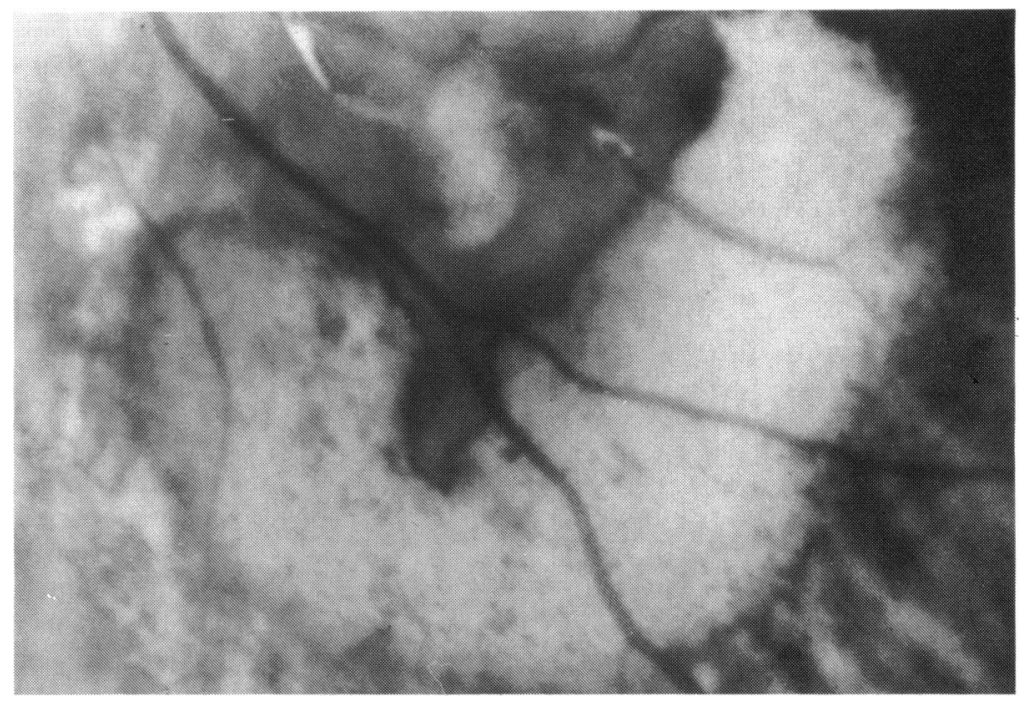

no significant aneurysmal leakage developed, and the vascular obstruction quickly became well compensated.

In our series retinal MAs occurred in 14 females and five males, showing a statistically very significant . female predominance (approximately 3:1). They were unilateral in $90 \%$ and bilateral in $10 \%$; single in $65 \%$ and multiple in $35 \%$ (Table 4 ). Aneurysms occurred on the superior temporal artery in $70 \%$ of the cases, on the inferior temporal artery in $35 \%$, and on the superior nasal artery in $10 \%$. None occurred on the inferior nasal artery. Each eye was affected with equal frequency (Table 4).

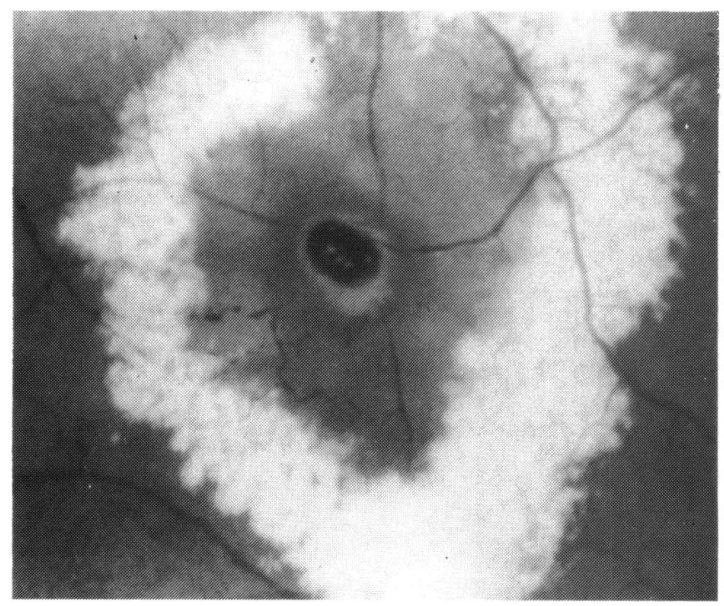

Fig. 6 Blow-out MA in a wide ring of retinal oedema encompassed by a solid ring of exudate. The perianeurysmal oedema is exaggerated by recent phototherapy between the $M A$ and exudative ring.
Group I MAs were equally divided between superior and inferior temporal arteries. Group II MAs showed a definite predisposition towards the superior temporal artery as against the $(10: 3)$ inferior temporal artery (Table 5).

Retinal MAs mainly affect the older population (age range 56 to 73 years), most of whom suffer from hypertension as well as other systemic cardiovascular

Table 3 Systemic disease associated with retinal MA

\begin{tabular}{llr}
\hline No. & Systemic disease & $\%$ \\
\hline 9 & Hypertension & 45 \\
6 & Cardiovascular disease & 30 \\
2 & Rheumatoid arthritis & 10 \\
1 & Diabetes & 5 \\
1 & Anaemia & 5 \\
1 & Raised sedimentation rate & 5 \\
\hline
\end{tabular}

Table 4 Occurrence of retinal $M A$ relating to sex, laterality, and number

\begin{tabular}{lrc}
\hline & No. & $\%$ \\
\hline Male & 5 & 30 \\
Female & 14 & 70 \\
Unilateral & 17 & 90 \\
Bilateral & 2 & 10 \\
Single & 13 & 65 \\
Multiple & 6 & 35 \\
\hline
\end{tabular}

Table 5 Site and distribution of retinal $M A$

\begin{tabular}{lrr}
\hline & No. & $\%$ \\
\hline Superior temporal artery & 14 & 60 \\
Inferior temporal artery & 8 & 35 \\
Superior nasal artery & 1 & 5 \\
\hline
\end{tabular}




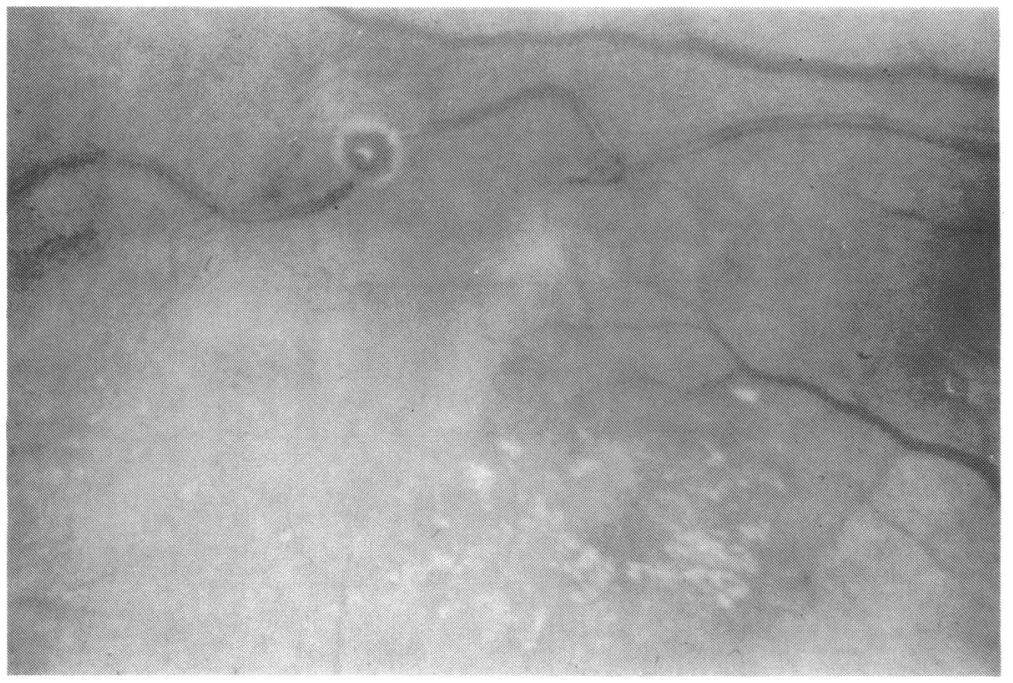

Fig. 7 In this case the haloed $M A$ was not encompassed by exudates; these were seen only at a distance, near the macula.

disease. ${ }^{45}$ Rheumatoid arthritis, diabetes, cerebrovascular disease and anaemia were also encountered (Table 3).

\section{Discussion}

Two classical types of aneurysm, saccular and fusiform, affect the retinal arteries. Fichte et al. ${ }^{3}$ preferred the terms 'blow-out' and 'cuffed'. The blow-out type in general was more prone to bleed (Fig. 8). Certainly this holds true for MAs on the first three orders of the retinal arterial tree, but large blow-outs on smaller arterioles may be exudative.

The haemorrhagic tendency of blow-out MAs may arise owing to the relatively easy perforation of a thin, stretched aneurysmal sac, especially where the perfusion pressure is high, as in the first three orders of arterioles. The lower perfusion pressure in MAs on smaller vessels may predispose these lesions to exudation.

Group II MAs on the first three orders of the retinal tree tended to be of the fusiform (cuffed) variety. It is possible that this configuration encourages leakage of plasma derivatives but resists frank perforation (Fig. 9).

In group I MAs, where the haemorrhage is primarily preretinal, it is likely that the arterial rupture has occurred on the vitreal side of the MA. Posterior or posterolateral rupture of the aneurysm probably leads to intra- and subretinal haemorrhage.

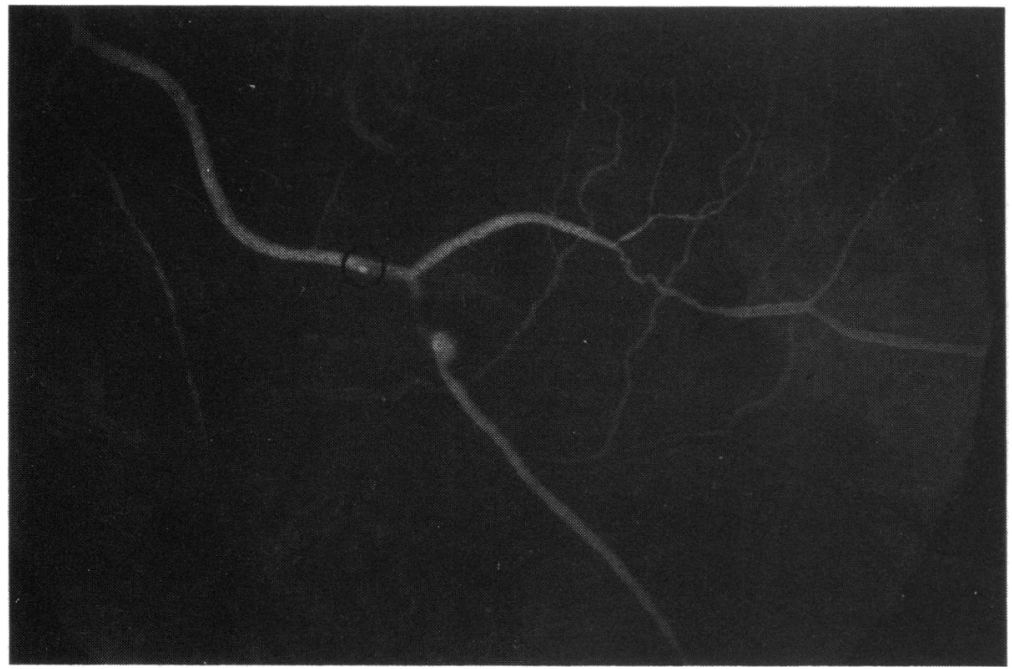

Fig. 8 Eight months after haemorrhage shown in Fig. 1 . Angiogram is arterial phase displaying $M A$ adjacent to first bifurcation of inferior temporal artery (ITA). Note circumscribed circle of hyperfluorescence apparently within the arterial lumen just proximal to the second arterial bifurcation (circle). The latter may indicate endothelial damage as a precursor to arterial wall decompensation. ${ }^{2}$ 
Fig. 9 MA with halo, indicating gross thickening of the aneurysmal sac wall centred in a florid circinate ring of exudate extending remotely from the aneurysm to reach the macular area.

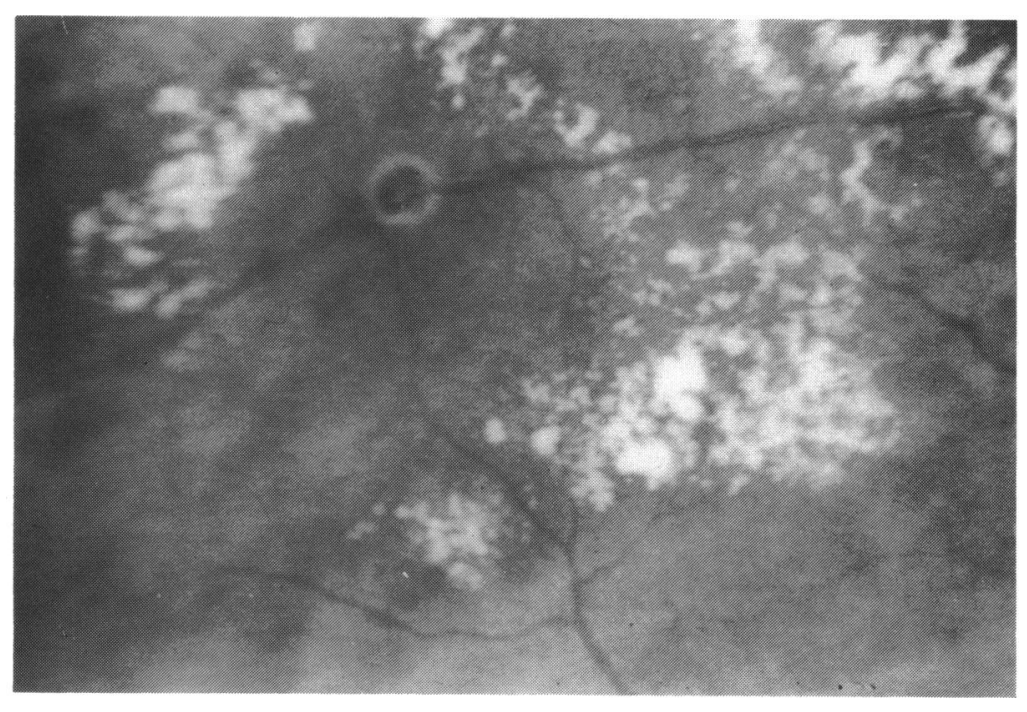

Large aneurysmal dehiscences produce harmorrhage at all levels (Fig. 10). Patients with acute haemorrhagic MAs have a better visual prognosis. Of the eight cases only one patient failed to regain useful central vision (Table 1, case 7). This patient was treated initially by perianeurysmal laser coagulation without any energy being applied directly to the MA. This seemed to close the MA, and no further treatment was given. She unfortunately bled from a

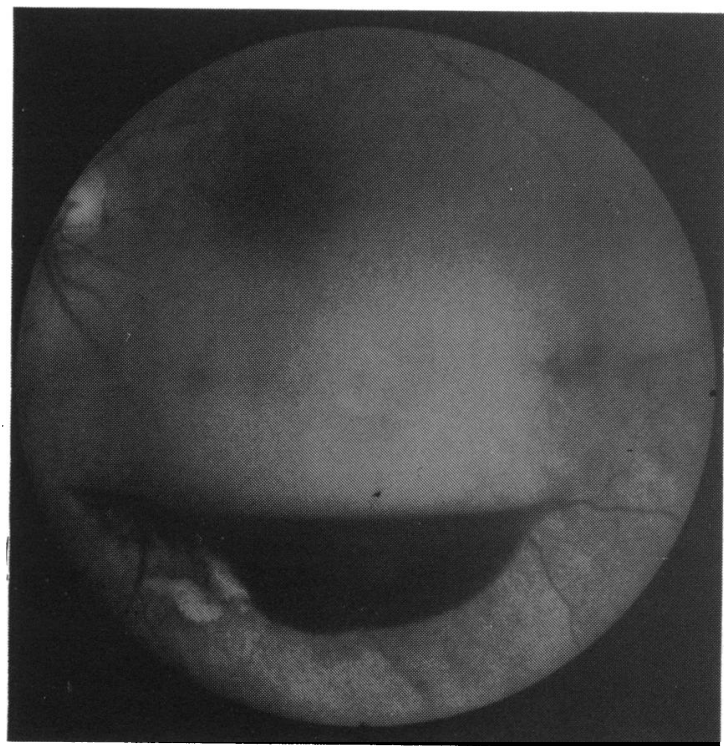

Fig. 10 Haemorrhage at all levels revealing an $M A$ with surrounding exudate; below that there are pigment clumping and subretinal scarring on the nasal side of the preretinal haemorrhage. second MA which formed distal to the original lesion on the superior temporal artery. This led to severe macular destruction and loss of central vision. Of the remaining seven patients (two requiring phototherapy) six regained central vision of $6 / 24$ or better after being reduced to levels of acuity varying from perception of light to $6 / 36$. One patient had a fall in vision by one line on the Snellen chart from 6/9 to 6/12 after a haemorrhage from a peripheral MA and subsequent degenerative macular changes.

Complete resorption of the haemorrhage occurred two to three months before the best visual acuity was achieved. A characteristic yellowish-grey plaque, akin to a disciform macular scar, subsequently developed at the site of intra- and subretinal bleeding (Figs. 1 and 5). The plaque is seen adjacent to the MA and is generally saddle-shaped, with the aneurysm in the saddle. Interestingly, the saddle was invariably inferior to the MA except in case 8 (Table 1), where the MA was sited on the superior temporal artery and the saddle was above the MA. If the plaque involved the macula (case 7, Table 1), central vision never recovered.

These plaques may evolve after a posterior aneurysmal rupture produces a dissecting haemorrhage and layer of blood superficial to the retinal pigment epithelium. The demarcation of this plaquelike scar with irregular pigmentation could then result from haemorrhagic penetration of the retinal pigment epithelium. The resultant scarring process is dissimilar from a disciform scar, because then there is neovascular penetration of Bruch's membrane.

In group II cases exudation was demonstrated by fluorescein angiography in the form of loss of vascular barrier to the dye, staining of the wall of the 


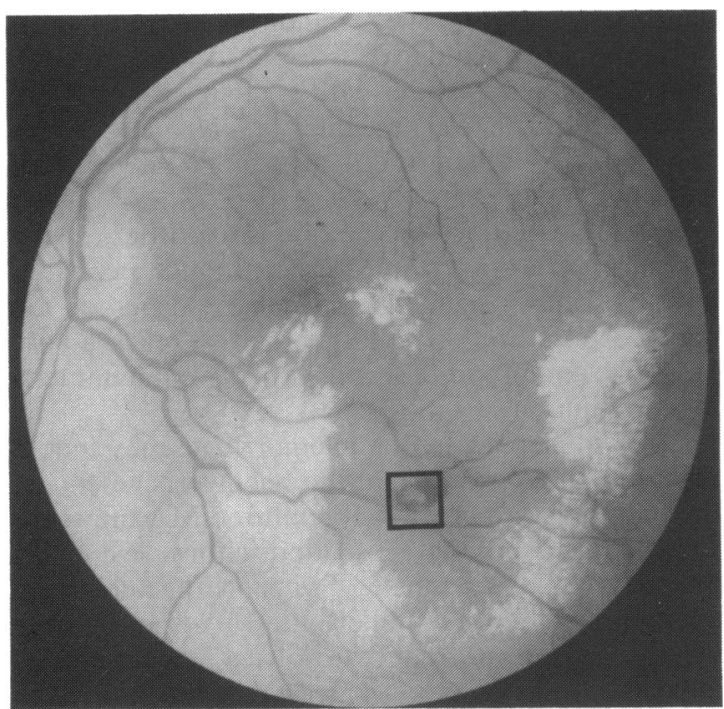

Fig. 11 A large blow-out MA within a venous bifurcation close to the main trunk of the ITA (square). Analysis of the fluorescein angiogram suggests the MA lies on a small radicle of the ITA. Encompassing the MA is a solid ring of exudate with intraretinal haemorrhage. Note bilobed dilation of ITA proximal to the origin of the feeder radicle to the $M A$.

aneurysm, and late leakage of the dye into the retina. In addition there is dilation and leakage from the capillary bed surrounding the dilated segment of the arteriole.

The occurrence of exudates at the macular area away from the offending vessel is not unique to retinal MA, as it occurs in other retinal vascular anomalies such as Coats' disease, Leber's miliary aneurysms, and von Hippel-Lindau diseases.

Exudates affecting the macular region, secondary to MA, occurred even when the MA was not in proximity to the macula, and we found that these cases had the worst prognosis whether treated or not (Fig. 11). Perianeurysmal exudates alone did not affect vision unless they extended to the macula. The distal circinate ring, in general, had a favourable prognosis and was absorbed if the aneurysm was treated.

In the group II cases three of 11 showed improvement in visual acuity ranging between two and three lines of the Snellen chart. The remaining eight showed no deterioration of their visual acuity. Six of these received phototherapy-three with xenon arc alone, one with argon laser alone, and two with xenon and argon.

Other signs were noted by previous workers, ${ }^{a-\infty}$ namely, kinking at the site of the resolved MA, arteriolar constriction proximal to the MA, and arterial sheathing distal to the MA. These changes were also noted in our series. Postaneurysmal arterial closure is rarely seen, but arterial stenosis and sheathing are common findings. Post haemorrhagic sheathing of the parent vessel was commonly encountered, as was extreme variation in the calibre of arteries harbouring MA. The kinked appearance at the site of the resolved MA may result from fibrotic changes in the media of the arterial wall causing traction on the adventitial portion in that segment, leading to shortening of the artery. Counterforces between the fibrous contracture and the tensile strength of the adventitial attachments to the internal limiting membrane of the retina will lead to the appearance of the Z-shaped kink (Fig. 3). The same appearance was seen in MAs which were directly photocoagulated, presumably due to a similar mechanism, though no arterial perforation or bleeding occurred.

\section{RESPONSE TO PHOTOTHERAPY}

No immediate or late complications of photocoagulation were noted. In particular there was no case of bleeding either at the time of treatment or subsequently.

Nine patients (three of eight group I and six of 11 group II) were treated with phototherapy; one of these patients had bilateral MAs and accordingly 10 eyes received treatment. Argon laser was used in five patients, one receiving treatment to both eyes. Five patients had xenon coagulation, one patient being treated with both xenon and argon. In all cases but one phototherapy direct to the MA was given, the exception being case 7 , group I.

In the last case the aneurysm subsided after preliminary perianeurysmal phototherapy, but it is worth noting that this patient developed a further aneurysm distal to the first along the same artery, with subsequent loss of central vision due to severe haemorrhage from the second aneurysm. The question remains whether direct phototherapy to the first MA may have forestalled the formation of the second aneurysm. In the remaining nine eyes the MA was directly treated in the first instance except in two cases where the MA was particularly large, and preliminary perianeurysmal treatment was thought to be necessary. In one case (case 4, group II) closure of the MA proved especially troublesome and in retrospect earlier phototherapy might have been advisable. No bleeding from direct photocoagulation of the MAs was encountered.

Of the 10 eyes treated two showed improvement in visual acuity (counting fingers to $6 / 24$, perception of light to 6/18). These were cases presenting with vitreous haemorrhage in which the responsible MA was closed to forestall rebleeding or halt progressive exudation. Seven eyes showed no alteration in visual 
acuity and were treated to prevent macular involvement in chronic MA-related exudation. One patient lost central vision from recurrent bleeding.

Of the remaining 11 eyes in the series eight were simply observed and three were treated with clofibrate. Five of the observed eyes presented with a ruptured MA (group I), all of which spontaneously formed a stable Z-shaped kink at the site of the former MA. Four of these recovered good central vision $(6 / 60$ to $6 / 9,1 / 60$ to $6 / 12,6 / 36$ to $6 / 9,6 / 60$ to $6 / 12$ ), and a fifth lost a single line on the Snellen chart (6/9 to 6/12) due to the onset of degenerative changes at the macula. A sixth observed case (case 5 , group I) had an asymptomatic MA in the contralateral eye. The remaining two observed eyes contained MAs secondary to arteriovenous occlusions. One of these achieved recovery of macular function (6/12 to 6/6) after a collateral circulation to the vascular obstruction had been established. The other (case 10, Table 2) had significant macular oedema and exudate and was awaiting phototherapy when she died.

Three group II eyes were treated with clofibrate. Two improved $(6 / 24$ to $6 / 9,6 / 18$ to $6 / 9)$, the latter (case 5, Table 2) with closure of the aneurysm and complete clearing of the exudate, the former with diminution of the exudate (case 3, Table 2). In the other case (case 1, Table 2) vision was unchanged and there was no increase in the volume of exudate. These three cases were seen early in our series and if seen later would simply have been observed and subjected to coagulation only if indicated.

Our indications for advising photocoagulation in retinal MA now are as follows:

(1) Recurrent bleeding or the threat of such. MA presenting only with haemorrhage should be observed initially and treatment offered only if decompensation of the MA continues without stable aneurysmal closure. Resolved MAs require no treatment.

(2) Exudates involving the macular area, since no time limit is known for the development of permanent damage to central visual function. Lewis et al. ${ }^{7}$ reported that direct treatment of MAs accelerated the rate of absorption of exudates with speedy recovery of visual function. It seems necessary to seal the MA directly to bring about immediate reduction in formation of exudates. As long as the MA is perfused exudates will continue to form, and we consider that direct phototherapy is a safe method of stemming aneurysmal leakage quickly and inducing closure of the MA.

\section{CONCLUSION}

Retinal MAs are probably commoner than is realised. They may confront the ophthalmologist in one of three ways: (1) sudden loss of vision due to haemorrhage (group I); (2) minor visual disturbances reflecting an exudative response (group II); (3) symptomless-either with exudates where there is no direct or indirect macular involvement or as a resolved MA in the form of a Z-shaped kink with or without plaque.

Retinal MAs arise on the first three orders of the retinal arterial tree in most cases, though occasionally they may originate on small branches of the major arterial tree. The latter tend to reach a very large size and promote severe exudative phenomena in the retina.

'Slow to clear' vitreous haemorrhage may hide a ruptured aneurysm. When the haemorrhage clears, the only sign of a previously ruptured MA may be a vessel kink overlying a pseudodisciform lesion. In this way MAs as a cause of vitreous haemorrhage may go unrecognised. Where the MA is still visible when the haemorrhage resolves, treatment is indicated, as the risk of recurrent haemorrhage and/or exudation is high. A Z-shaped kink indicates a resolved and stable MA and does not require treatment. Treatment should also be given when macular function is threatened by leakage and exudation from the weakened wall of the MA and dilated surrounding capillaries.

We favour phototherapy direct to the MA either with xenon arc or laser, and we have observed no untoward sequelae. We have not encountered bleeding during or after treatment, as have others, 56910 though we accept that the risk of haemorrhage is probably greater with the argon laser. Direct treatment speeds the rate of absorption of perianeurysmal exudates and seals the MA itself. The question of direct or indirect treatment of MAs, however, remains controversial, ${ }^{15611}$ and further study and follow-up are necessary.

We acknowledge with thanks the expert and industrious help of Mr Michael Strachan, medical photographer, and the following consultants who were kind enough to refer cases: Mr Claud Sharp, Mr David Allen, Mr R W Allchin, Mr Neil Manson.

\section{References}

1 Campbell FP. Coats' disease and congenital vascular retinopathy. Trans Am Ophthalmol Soc 1976; 74: 366-424.

2 Robertson DM. Macroaneurysms of the retinal arteries. Ophthalmology (Rochester) 1973; 77: 55-67.

3 Fichte C, Streeten BW, Friedman AH. Histopathological study of retinal arterial aneurysm. Am J Ophthalmol 1978; 85: 509-18.

4 François J. Acquired macroaneurysms of the retinal arteries. Int Ophthalmol 1979; i: 153-61.

5 Nadel AJ, Gupta KK. Macroaneurysms of the retinal arteries. Arch Ophthalmol 1976; 94: 1092-6.

6 Palestine AG, Robertson DM, Goldstein B. Macroaneurysms of the retinal arteries. Am J Ophthalmol 1982; 93: 164-71.

7 Lewis RA, Norton EWD, Gass JDM. Acquired arterial macroaneurysms of the retinal arteries. Br J Ophthalmol 1976; 60: $21-30$ 
8 Cleary PE, Kohner EM, Hamilton AM, Bird AC. Retinal macroaneurysms. BrJ Ophthalmol 1975; 59: 355-61.

9 Hudomel J, Imre G. Retinal macroaneurysms. Acta Ophthalmol (Kbh) 1973; 51: 633-8.
10 Shults WT, Swan KC. Pulsatile aneurysms of the retinal arterial trec. Am J Ophthalmol 1974; 77: 304-9.

11 Nouhuys FV. Deutman AF. Argon laser treatment of retinal macroaneurysms. Int Ophthalmol 1980; 1: 45-53.

Accepted for publication 28 March 1985. 